\title{
Global existence and attractors for the two-dimensional Burgers-Ginzburg- Landau equations
}

\author{
Changhong Guo ${ }^{\mathrm{a}}$, Shaomei Fang ${ }^{\mathrm{b}, *}$ \\ a School of Management, Guangdong University of Technology, Guangzhou 510520, P. R. China. \\ ${ }^{b}$ Department of Mathematics, South China Agricultural University, Guangzhou 510640, P. R. China. \\ Communicated by G. Marino
}

\begin{abstract}
This paper investigates the periodic initial value problem for the two-dimensional Burgers-Ginzburg-Landau (2D BurgersGL) equations, which can be derived from the so-called modulated modulation equations (MME) that govern the dynamics of the modulated amplitudes of some periodic critical modes. The well-posedness of the solutions and the global attractors for the 2D Burgers-GL equations are obtained via delicate a priori estimates, the Galerkin method, and operator semigroup method. (C)2017 All rights reserved.
\end{abstract}

Keywords: 2D Burgers-GL equations, well-posedness, global attractors, a priori estimates. 2010 MSC: 35D35, 35B41, 35Q56.

\section{Introduction}

In this paper, we consider the following two-dimensional Burgers-Ginzburg-Landau (2D Burgers-GL) equations

$$
\begin{aligned}
& v_{1 \mathrm{t}}=\alpha v_{1 x x}+\alpha v_{1 y y}+\beta v_{1} v_{1 x}+\beta v_{2} v_{1 y}+\gamma\left(|A|^{2}\right)_{x}, \\
& v_{2 x}=v_{1 y} \\
& A_{t}=\mu_{0} A+\left(\mu_{1}+i \mu_{2}\right)\left(A_{x x}+A_{y y}\right)+s_{1}\left(v_{1}^{2}+v_{2}^{2}\right)^{\frac{1}{2}} A-\left(s_{2}|A|^{2}+s_{3}\left(v_{1}^{2}+v_{2}^{2}\right)\right) A,
\end{aligned}
$$

where the velocity components $v_{1}=v_{1}(x, y, t)$ and $v_{2}=v_{2}(x, y, t)$ are real-valued functions, and $A=$ $A(x, y, t)$ is the complex-valued function. $(x, y) \in \Omega, \Omega$ is a bounded domain in two-dimensional real Euclidean space. The coefficients $\alpha, \beta, \gamma, \mu_{0}, \mu_{1}$, and $\mu_{2}$ are real constants, while $s_{1}, s_{2}$, and $s_{3}$ are complex constants. Similar to the derivation by the Cole-Hopf transformation in [17], the 2D Burgers-GL equations (1.1)-(1.3) can be rewritten in another coupled form as

$$
\begin{aligned}
v_{\mathrm{t}} & =\alpha \Delta v+\beta(v \cdot \nabla) v+\gamma \nabla\left(|A|^{2}\right), \\
A_{\mathrm{t}} & =\mu_{0} \mathrm{~A}+\left(\mu_{1}+i \mu_{2}\right) \Delta \mathrm{A}+\mathrm{s}_{1}|v| A-\left(\mathrm{s}_{2}|A|^{2}+s_{3}|v|^{2}\right) A,
\end{aligned}
$$

\footnotetext{
*Corresponding author

Email addresses: cmchguo@gdut.edu.cn (Changhong Guo), fangsm90@163.com (Shaomei Fang)
} doi:10.22436/jnsa.010.06.27 
where $v=\left\{v_{1}, v_{2}\right\},|v|=\sqrt{v_{1}^{2}+v_{2}^{2}}$.

The Burgers-GL equations (1.4)-(1.5) in one-dimensional can be derived from the so-called modulated modulation equations (MME) deduced by Harten [19]. In his study of the Ginzburg-Landau equation as a modulation equation for the amplitude of a periodic critical mode in various applications, he found that there is the less well-known possibility of an instability of non-sideband type for the family of periodic solution of the Ginzburg-Landau equation besides the Eckhaus' instability. And then he deduced three so-called MME under the different coefficients of the original Ginzburg-Landau equation which consist of the critical mode(s) with an amplitude modulated in space and time. One of the three MMEs is a real gradient system of Kuramoto-Shivasinsky type derived with multiple scaling techniques [14]. Another is a perturbed Korteweg-de-Vries derived for an Eckhaus' instability by Bernoff [3]. The last one seems to be a new result and has the form of Burgers equation coupled to the Ginzburg-Landau equation, which is the Burgers-GL equations (1.4)-(1.5) in one-dimensional.

If there is no coupling term with $A$ in Eq. (1.4), then Eq. (1.4) would be the well-known twodimensional Burgers equations [17], which is transformed by the Cole-Hopf transformation and is an integrable generalization of the well-known Burgers equation [4]. Some researches have been done in physical study and the mathematical analysis of the two-dimensional Burgers equations, such as the stationary solutions [13], the exact solutions [1], the numerical solutions [2, 20], and so on. Meanwhile, if there is no coupling terms with $v$ in Eq. (1.5), the Eq. (1.5) reduces to the well-known CGL equation, which is considered as the generic modulation equation near the onset of instabilities in non-equilibrium fluid dynamical systems, as well as in the theory of phase transitions and superconductivity [15, 16]. For some other results involved with the CGL equation, see $[6,7]$ and reference therein. However, little progress has been obtained for the coupled Burgers-GL equations (1.4)-(1.5), Since Guo and Huang studied the well-posedness and global attractors for one-dimensional Burgers-GL equations in [8] and [9]. Afterwards Huang continued to study the one-dimensional Burgers-GL equations in discrete version by the finite difference method in spatial direction [11] and that with non-homogeneous term by the LeraySchauder fixed point theorem [12]. Subsequent to previous work in one-dimension Burgers-GL equations, in this paper we are further going to consider the 2D Burgers-GL equations (1.1)-(1.3), with the periodic boundary conditions

$$
\begin{aligned}
v(x+L, y, t) & =v(x, y, t), \quad v(x, y+L, t)=v(x, y, t), \\
A(x+L, y, t) & =A(x, y, t), \quad A(x, y+L, t)=A(x, y, t), \\
\int_{\Omega} v(x, t) d x & =0, \quad t>0,
\end{aligned}
$$

and the initial conditions

$$
v(x, y, 0)=v_{0}(x, y), \quad A(x, y, 0)=A_{0}(x, y),
$$

where $L>0$ is the period, and $v_{0}(x, y)$ and $A_{0}(x, y)$ are given functions.

In what follows, we are going to study the well-posedness and global attractors for the periodic initial value problem via delicate a priori estimates and operator semigroup method. In our argument, we set $s_{2}=s_{2 r}+i s_{2 i}$, where $s_{2 r}$ and $s_{2 i}$ are the real part and imaginary part of $s_{2}$, respectively. And we make some basic assumptions as

$$
\alpha>0, \quad \mu_{0}>0, \quad \mu_{1}>0, \quad s_{2 r}>0, \quad\left|s_{2 i}\right|<\sqrt{3} s_{2 r}, \quad \operatorname{Re}\left(s_{3}\right)>0 .
$$

The rest of paper is organized as follows. In Section 2, we briefly give some notations and preliminaries. In Section 3, we establish a prior estimates for the solutions of the periodic initial value problem (1.4)-(1.9). In Section 4, the well-posedness for the 2D Burgers-GL equations are obtained via the Galerkin method and so-called continuity method. In the last Section 5, the existence of the global attractors are obtained by constructing the uniform a priori estimates in time. 


\section{Notations and preliminaries}

For the mathematical setting, we introduce several function spaces and notations. We denote

$$
\begin{aligned}
\mathbf{L}^{\mathfrak{p}}(\Omega) & =\left\{v=\left\{v_{1}, v_{2}\right\} \mid v_{1} \in \mathrm{L}^{\mathbf{p}}(\Omega), v_{2} \in \mathrm{L}^{\mathbf{p}}(\Omega)\right\}, \\
\mathbf{W}^{\mathrm{k}, \mathrm{p}}(\Omega) & =\left\{v=\left\{v_{1}, v_{2}\right\} \mid v_{1} \in \mathbf{W}^{\mathrm{k}, \mathrm{p}}(\Omega), v_{2} \in \mathbf{W}^{\mathrm{k}, \mathrm{p}}(\Omega)\right\},
\end{aligned}
$$

where $\mathrm{L}^{\mathrm{p}}(\Omega)$ and $\mathrm{W}^{\mathrm{k}, \mathrm{p}}(\Omega)\left(\mathrm{k} \in \mathrm{N}^{+}, 1 \leqslant \mathrm{p} \leqslant \infty\right)$ are the usual Lebesgue and Sobolev spaces, respectively. When $p=2$, we denote $\mathbf{L}^{2}=\mathbf{L}^{p}(\Omega)$ and $\mathbf{H}^{k}=\mathbf{W}^{k, 2}(\Omega)$ for simplicity. These two spaces are equipped with the following inner products and norms:

$$
\begin{aligned}
& (v, u)=\sum_{i=1}^{2}\left(v_{i}, u_{i}\right)=\sum_{i=1}^{2} \int_{\Omega} v_{i} u_{i} d x, \\
& \|v\|^{2}=(v, v), \quad\|v\|_{H^{k}}=\left(\sum_{|l| \leqslant k}\left\|D^{l} v\right\|^{2}\right)^{\frac{1}{2}} .
\end{aligned}
$$

Meanwhile, we introduce complex Sobolev spaces. In general, we denote by $X, y, \ldots$, the complexified space of a function $X, Y, \cdots$. For example, $\mathcal{L}^{2}$ and $\mathcal{H}^{k}$ are the complexified spaces of $L^{2}(\Omega)$ and $H^{k}(\Omega)$, respectively. If $A \in \mathcal{L}^{2}, B \in \mathcal{L}^{2}$, we define

$$
(A, B)=\int_{\Omega} A \bar{B} d x, \quad\|A\|^{2}=(A, A), \quad\|A\|_{\mathcal{H}^{k}}=\left(\sum_{|l| \leqslant k}\left\|D^{l} A\right\|^{2}\right)^{\frac{1}{2}},
$$

where $\bar{B}$ denotes the complex conjugate of $B$. Furthermore, $X_{\text {per }}$ denotes the set of all periodic functions that are contained in the space $X$.

Without any ambiguity, we denote a generic positive constant by $\mathrm{C}$ which may vary from line to line.

Lemma 2.1 (Gagliardo-Nirenberg inequality, [5]). Let $\Omega$ be a bounded domain with $\partial \Omega$ in $\mathrm{C}^{\mathrm{m}}$, and let $\mathrm{u}$ be any function in $\mathrm{W}^{\mathrm{m}, \mathrm{r}}(\Omega) \cap \mathrm{L}^{\mathrm{q}}(\Omega), 1 \leqslant \mathrm{q}, \mathrm{r} \leqslant \infty$. For any integer $\mathrm{j}, 0 \leqslant j<\mathrm{m}$, and for any number $\mathrm{a}$ in the interval $\mathrm{j} / \mathrm{m} \leqslant \mathrm{a} \leqslant 1$, set

$$
\frac{1}{p}=\frac{j}{n}+a\left(\frac{1}{r}-\frac{m}{n}\right)+(1-a) \frac{1}{q} .
$$

If $\mathrm{m}-\mathrm{j}-\mathrm{n} / \mathrm{r}$ is not a nonnegative integer, then

$$
\left\|D^{j} u\right\|_{L^{p}} \leqslant C\|u\|_{W^{m, r}}^{a}\|u\|_{L^{q}}^{1-a} .
$$

If $m-j-n / r$ is a nonnegative integer, then (2.1) holds for $a=j / m$. The constant $C$ depends only on $\Omega, r, q, j, a$.

In the sequel, we will use the following inequalities for two-dimensional equations as the specific cases of the Gagliardo-Nirenberg inequality:

$$
\begin{aligned}
\text { \%labelGNYoujieinequality } 2\left\|D^{j} u\right\|_{L^{\infty}} \leqslant C\|u\|_{H^{m}}^{a}\|u\|^{1-a}, & m a=j+1, \\
\left\|D^{j} u\right\|_{L^{2}} \leqslant C\|u\|_{H^{m}}^{a}\|u\|^{1-a}, & m a=j, \\
\left\|D^{j} u\right\|_{L^{4}} \leqslant C\|u\|_{H^{m}}^{a}\|u\|^{1-a}, & m a=j+1 / 2 .
\end{aligned}
$$

Lemma 2.2 (The uniform Gronwall lemma, [18]). Let $\mathrm{g}, \mathrm{h}, \mathrm{y}$ be three positive locally integrable functions on $\left[\mathrm{t}_{0}, \infty\right)$, such that $\mathrm{y}^{\prime}$ is locally integrable on $\left[\mathrm{t}_{0}, \infty\right)$, and which satisfy

$$
\begin{aligned}
& \frac{d y}{d t} \leqslant g y+h, \quad \text { for } t \geqslant t_{0}, \\
& \int_{t}^{t+r} g(s) d s \leqslant a_{1}, \quad \int_{t}^{t+r} h(s) d s \leqslant a_{2}, \quad \int_{t}^{t+r} y(s) d s \leqslant a_{3}, \text { for } t \geqslant t_{0},
\end{aligned}
$$


where $r, a_{1}, a_{2}, a_{3}$ are positive constants. Then

$$
y(t+r) \leqslant\left(\frac{a_{3}}{r}+a_{2}\right) \exp \left(a_{1}\right), \text { for } t \geqslant t_{0}
$$

\section{A priori estimates}

In this section, we derive some a priori estimates for the solutions of the periodic initial value problem (1.4)-(1.9). Firstly we have

Lemma 3.1. Assume $v_{0}(x) \in \mathbf{L}_{\text {per }}^{2}(\Omega), A_{0}(x) \in \mathcal{L}_{\text {per }}^{2}(\Omega)$, and the assumptions (1.10) hold. Then for the solutions of the problem (1.4)-(1.9), we have

$$
\begin{aligned}
& \|v\|^{2} \leqslant\left(\left\|v_{0}\right\|^{2}+\frac{|\gamma|^{2}}{s_{2 r}}\left\|A_{0}\right\|^{2}\right) e^{-\theta t}+\frac{2|\gamma|^{2} C_{2}}{\alpha \theta s_{2 r}}\left(1-e^{-\theta t}\right), \\
& \|A\|^{2} \leqslant\left\|A_{0}\right\|^{2} e^{-t}+C_{2}\left(1-e^{-t}\right)
\end{aligned}
$$

and

$$
\limsup _{t \rightarrow \infty}\left(\|v\|^{2}+\|A\|^{2}\right) \leqslant \frac{2|\gamma|^{2} C_{2}}{\alpha \theta s_{2 r}}+C_{2}=\rho_{0}^{2}
$$

Furthermore, we have

$$
\int_{t}^{t+r}\|\nabla v\|^{2} \mathrm{ds} \leqslant \frac{|\gamma|^{2}}{\alpha^{2} C_{3}}\left(C_{2} r+\left\|A_{0}\right\|^{2}+C_{2}\right)+\frac{1}{\alpha}\left(\left\|v_{0}\right\|^{2}+\frac{|\gamma|^{2}}{s_{2 r}}\left\|A_{0}\right\|^{2}\right)+\frac{2|\gamma|^{2} C_{2}}{\alpha^{2} \theta s_{2 r}},
$$

and

$$
\int_{t}^{t+r}\|\nabla A\|^{2} \mathrm{~d} s+\int_{t}^{t+r} \int_{\Omega}|A|^{4} \mathrm{~d} x \mathrm{~d} s+\int_{t}^{t+r} \int_{\Omega}|v|^{2}|A|^{2} \mathrm{~d} x \mathrm{~d} s \leqslant \frac{1}{C_{3}}\left(C_{2} r+\left\|A_{0}\right\|^{2}+C_{2}\right)
$$

for all $\mathrm{r}>0$, where $\theta, \mathrm{C}_{2}$, and $\mathrm{C}_{3}$ are positive constants depending on the known parameters.

Proof. Multiplying (1.5) by $\bar{A}$, integrating with respect to $x$ over $\Omega$ and taking the real part, we obtain

$$
\frac{1}{2} \frac{\mathrm{d}}{\mathrm{dt}}\|\mathrm{A}\|^{2}+\mu_{1}\|\nabla \mathrm{A}\|^{2}+s_{2 \mathrm{r}} \int_{\Omega}|A|^{4} \mathrm{~d} x+\operatorname{Re}\left(s_{3}\right) \int_{\Omega}|v|^{2}|A|^{2} \mathrm{~d} x=\mu_{0}\|A\|^{2}+\operatorname{Re}\left(s_{1}\right) \int_{\Omega}|v \| A|^{2} \mathrm{~d} x .
$$

According to the Hölder's inequality and Yong's inequality with $\varepsilon$, we have

$$
\begin{aligned}
\mu_{0}\|A\|^{2}+\operatorname{Re}\left(s_{1}\right) \int_{\Omega}|v \| A|^{2} \mathrm{~d} x & \leqslant \mu_{0}\|\mathrm{~A}\|^{2}+\left|s_{1}\right|\left(\int_{\Omega}|v|^{2}|A|^{2} \mathrm{~d} x\right)^{\frac{1}{2}}\|A\| \\
& \leqslant \frac{1}{2} \operatorname{Re}\left(s_{3}\right) \int_{\Omega}|v|^{2}|A|^{2} \mathrm{~d} x+\left(\frac{\left|s_{1}\right|^{2}}{2 \operatorname{Re}\left(s_{3}\right)}+\mu_{0}\right)\|A\|^{2} \\
& \leqslant \frac{1}{2} \operatorname{Re}\left(s_{3}\right) \int_{\Omega}|v|^{2}|A|^{2} \mathrm{~d} x+\frac{1}{2} s_{2 r} \int_{\Omega}|A|^{4} \mathrm{~d} x+\mathrm{C}_{1}
\end{aligned}
$$

where $C_{1}$ is a positive constant depending on $\mu_{0}, s_{1}, s_{2}, s_{3}$, and $|\Omega|$, the two-dimensional measure of $\Omega$. Combining (3.5) and (3.6) together yields that

$$
\frac{\mathrm{d}}{\mathrm{dt}}\|\mathrm{A}\|^{2}+2 \mu_{1}\|\nabla A\|^{2}+s_{2 r} \int_{\Omega}|A|^{4} \mathrm{~d} x+\operatorname{Re}\left(s_{3}\right) \int_{\Omega}|v|^{2}|A|^{2} \mathrm{~d} x \leqslant \mathrm{C}_{1}
$$

Noticing that $\|A\|^{2} \leqslant \frac{1}{2} s_{2 r} \int_{\Omega}|A|^{4} \mathrm{~d} x+|\Omega| / 2 s_{2 r}$, we obtain

$$
\frac{d}{d t}\|A\|^{2}+\|A\|^{2}+2 \mu_{1}\|\nabla A\|^{2}+\frac{1}{2} s_{2 r} \int_{\Omega}|A|^{4} d x+\operatorname{Re}\left(s_{3}\right) \int_{\Omega}|v|^{2}|A|^{2} d x \leqslant C_{1}+\frac{|\Omega|}{4 s_{2 r}}=C_{2} .
$$


By the Gronwall's inequality, we have

$$
\|A\|^{2} \leqslant\left\|A_{0}\right\|^{2} e^{-t}+C_{2}\left(1-e^{-t}\right),
$$

which concludes (3.2).

Next, we take the inner product of (1.4) with $v$ in $\mathbf{L}^{2}(\Omega)$ to have

$$
\frac{1}{2} \frac{\mathrm{d}}{\mathrm{dt}}\|v\|^{2}+\alpha\|\nabla v\|^{2}=\beta \mathrm{b}(v, v, v)+\gamma \int_{\Omega} \nabla\left(|A|^{2}\right) v \mathrm{~d} x
$$

where

$$
\mathrm{b}(\mathrm{u}, v, w)=\int_{\Omega}\left(u_{1} v_{1 x} w_{1}+u_{2} v_{1 y} w_{1}+u_{1} v_{2 x} w_{2}+u_{2} v_{2 y} w_{2}\right) d x d y
$$

for $u=\left\{\mathfrak{u}_{1}, \mathfrak{u}_{2}\right\}, v=\left\{v_{1}, v_{2}\right\}$, and $\boldsymbol{w}=\left\{w_{1}, w_{2}\right\}$, whenever the integrals make sense. Obviously, there holds that $\mathrm{b}(v, v, w)=((v \cdot \nabla) v, w)$. Actually, the form $\mathrm{b}$ is trilinear continuous on $\mathbf{H}^{1}(\Omega)$. Generally, we have the following inequalities giving various continuity properties of $b(u, v, w)$ [18]

$$
|\mathrm{b}(\mathrm{u}, v, w)| \leqslant \mathrm{C}_{\mathrm{b}} \times\left\{\begin{array}{l}
\|\mathrm{u}\|^{\frac{1}{2}}\|\nabla \mathfrak{u}\|^{\frac{1}{2}}\|\nabla v\|^{\frac{1}{2}}\|\Delta v\|^{\frac{1}{2}}\|w\|, \\
\|\mathfrak{u}\|^{\frac{1}{2}}\|\Delta u\|^{\frac{1}{2}}\|\nabla v\|\|w\|, \\
\|\mathfrak{u}\|\|\nabla v\|\|w\|^{\frac{1}{2}}\|\Delta w\|^{\frac{1}{2}} \\
\|u\|^{\frac{1}{2}}\|\nabla u\|^{\frac{1}{2}}\|\nabla v\|\|w\|^{\frac{1}{2}}\|\nabla w\|^{\frac{1}{2}}
\end{array}\right.
$$

where $\mathrm{C}_{\mathrm{b}}>0$ is an appropriate constant.

First, according to the integration by parts and the Eq. (1.2), there holds

$$
\mathrm{b}(v, v, v)=0 .
$$

Second, we have

$$
\gamma \int_{\Omega} \nabla\left(|A|^{2}\right) v \mathrm{~d} x \leqslant|\gamma| \int_{\Omega}|A|^{2}|\nabla v| \mathrm{d} x \leqslant \frac{1}{2} \alpha\|\nabla v\|^{2}+\frac{|\gamma|^{2}}{2 \alpha} \int_{\Omega}|A|^{4} \mathrm{~d} x .
$$

Substituting (3.12) and (3.13) into (3.9), it follows

$$
\frac{\mathrm{d}}{\mathrm{dt}}\|v\|^{2}+\alpha\|\nabla v\|^{2} \leqslant \frac{|\gamma|^{2}}{\alpha} \int_{\Omega}|A|^{4} \mathrm{~d} x
$$

Under the condition $\int_{\Omega} v \mathrm{~d} x=0$, we have $\|v\| \leqslant \mathrm{C}_{*}\|\nabla v\|$ from the Poincaré's inequality. Then from (3.14) and multiplying $\frac{1}{2} s_{2 r}$ on both sides, there holds

$$
\frac{\mathrm{d}}{\mathrm{dt}}\left(\frac{1}{2} s_{2 \mathrm{r}}\|v\|^{2}\right)+\frac{\alpha s_{2 r}}{2 \mathrm{C}_{*}^{2}}\|v\|^{2} \leqslant \frac{|\gamma|^{2} s_{2 r}}{2 \alpha} \int_{\Omega}|A|^{4} \mathrm{~d} x
$$

Meanwhile from (3.7), we have

$$
\frac{\mathrm{d}}{\mathrm{dt}}\left(\frac{|\gamma|^{2}}{\alpha}\|\mathrm{A}\|^{2}\right)+\frac{|\gamma|^{2}}{\alpha}\|A\|^{2}+\frac{|\gamma|^{2} s_{2 r}}{2 \alpha} \int_{\Omega}|A|^{4} \mathrm{~d} x \leqslant \frac{|\gamma|^{2} \mathrm{C}_{2}}{\alpha} .
$$

Combining (3.15) and (3.16) together yields that

$$
\frac{\mathrm{d}}{\mathrm{dt}}\left(\frac{1}{2} s_{2 r}\|v\|^{2}+\frac{|\gamma|^{2}}{\alpha}\|\mathrm{A}\|^{2}\right)+\theta\left(\frac{1}{2} s_{2 r}\|v\|^{2}+\frac{|\gamma|^{2}}{\alpha}\|\mathrm{A}\|^{2}\right) \leqslant \frac{|\gamma|^{2} C_{2}}{\alpha},
$$

where $\theta=\min \left\{\frac{\alpha}{\mathrm{C}_{*}^{2}}, 1\right\}>0$. By the Gronwall's inequality, we have

$$
\|v\|^{2} \leqslant\left(\left\|v_{0}\right\|^{2}+\frac{|\gamma|^{2}}{s_{2 r}}\left\|A_{0}\right\|^{2}\right) e^{-\theta t}+\frac{2|\gamma|^{2} C_{2}}{\alpha \theta s_{2 r}}\left(1-e^{-\theta t}\right),
$$

which implies (3.1). Thus from (3.8) and (3.17), we have 


$$
\limsup _{t \rightarrow \infty}\left(\|v\|^{2}+\|A\|^{2}\right) \leqslant \frac{2|\gamma|^{2} C_{2}}{\alpha \theta s_{2 r}}+C_{2}=\rho_{0}^{2}
$$

We consider the space $E_{0}$ normed by $\|\psi\| E_{E_{0}}=\left\{\|v\|^{2}+\|A\|^{2}\right\}^{\frac{1}{2}}$, for all $\psi=\{v, A\}$. Thus we deduce from (3.8), (3.17), and (3.18) that the balls $\mathrm{B}_{\mathrm{E}_{0}}(0, \rho)$ of $\mathrm{E}_{0}$ centered at 0 of radius $\rho>\rho_{0}$ are positively invariants and are absorbing in $E_{0}$ for the semigroup $S(t)$. We choose $\rho_{0}^{\prime}>\rho_{0}$ and denote by $\mathcal{B}_{0}$ the ball $B_{E_{0}}\left(0, \rho_{0}^{\prime}\right)$. Any set $\mathcal{B}$ bounded in $E_{0}$ is included in a ball $B(0, R)$ of $E_{0}$. Then there holds $S(t) \mathcal{B} \subset \mathcal{B}_{0}$ for $t>t_{0}\left(\mathcal{B}, \rho_{0}^{\prime}\right)$, where

$$
t_{0}=\frac{1}{\min \{1, \theta\}} \ln \frac{2 R^{2}+\frac{|\gamma|^{2}}{s_{2 r}} R^{2}}{\left(\rho_{0}^{\prime}\right)^{2}-\rho_{0}^{2}} .
$$

Finally, we infer from (3.7) and (3.8), after integration in $t$, that

$$
\begin{aligned}
\int_{t}^{t+r}\|\nabla A\|^{2} \mathrm{~d} s+\int_{t}^{t+r} \int_{\Omega}|A|^{4} \mathrm{~d} x \mathrm{~d} s+\int_{t}^{t+r} \int_{\Omega}|v|^{2}|A|^{2} \mathrm{~d} x \mathrm{~d} s & \leqslant \frac{1}{\mathrm{C}_{3}}\left(\int_{t}^{\mathrm{t}+\mathrm{r}} \mathrm{C}_{2} \mathrm{~d} s+\|A(\mathrm{t})\|^{2}\right) \\
& \leqslant \frac{1}{\mathrm{C}_{3}}\left(\mathrm{C}_{2} \mathrm{r}+\left\|\mathrm{A}_{0}\right\|^{2}+\mathrm{C}_{2}\right),
\end{aligned}
$$

where $C_{3}=\min \left\{2 \mu_{1}, \frac{1}{2} s_{2 r}, \operatorname{Re}\left(s_{3}\right)\right\}$. This concludes (3.4). Meanwhile, integrating (3.14) in $t$ and combining (3.20), we have

$$
\begin{aligned}
\int_{t}^{t+r}\|\nabla v\|^{2} d s & \leqslant \frac{1}{\alpha}\left(\frac{|\gamma|^{2}}{\alpha} \int_{t}^{t+r} \int_{\Omega}|A|^{4} \mathrm{~d} x \mathrm{ds}+\|v(\mathrm{t})\|^{2}\right) \\
& \leqslant \frac{|\gamma|^{2}}{\alpha^{2} C_{3}}\left(C_{2} r+\left\|A_{0}\right\|^{2}+C_{2}\right)+\frac{1}{\alpha}\left(\left\|v_{0}\right\|^{2}+\frac{|\gamma|^{2}}{s_{2 r}}\left\|A_{0}\right\|^{2}\right)+\frac{2|\gamma|^{2} C_{2}}{\alpha^{2} \theta s_{2 r}},
\end{aligned}
$$

which concludes (3.3). Thus the proof of Lemma 3.1 is completed.

Lemma 3.2. Assume $v_{0}(x) \in \mathbf{H}_{\text {per }}^{1}(\Omega), A_{0}(x) \in \mathcal{H}_{\text {per }}^{1}(\Omega)$, and the conditions in Lemma 3.1 hold. Then for the solutions of the problem (1.4)-(1.9), we have

$$
\|\nabla v\|^{2}+\|\nabla A\|^{2} \leqslant\left(\frac{a_{3}}{r}+a_{2}\right) e^{a_{1}}, \quad \text { for } \quad t \geqslant t_{0}+r, \quad \forall r>0,
$$

where $\mathrm{a}_{1}, \mathrm{a}_{2}$, and $\mathrm{a}_{3}$ are positive constants.

Proof. Taking the inner product of (1.4) with $-\Delta v$ in $\mathbf{L}^{2}(\Omega)$, we have

$$
\frac{1}{2} \frac{\mathrm{d}}{\mathrm{dt}}\|\nabla v\|^{2}+\alpha\|\Delta v\|^{2}=-\beta \mathrm{b}(v, v, \Delta v)-\gamma\left(\nabla\left(|\mathrm{A}|^{2}\right), \Delta v\right) .
$$

Multiplying (1.5) by $-\Delta \overline{\mathrm{A}}$, integrating with respect to $x$ over $\Omega$ and taking the real part, we have

$$
\begin{aligned}
\frac{1}{2} \frac{\mathrm{d}}{\mathrm{dt}}\|\nabla \mathrm{A}\|^{2}+\mu_{1}\|\Delta \mathrm{A}\|^{2}= & \mu_{0}\|\nabla \mathrm{A}\|^{2}-\operatorname{Re}\left(s_{1} \int_{\Omega}|v| \mathrm{A} \Delta \overline{\mathrm{A}} \mathrm{d} x\right)+\operatorname{Re}\left(\left(s_{2 r}+i s_{2 i}\right) \int_{\Omega}|\mathrm{A}|^{2} \mathrm{~A} \Delta \overline{\mathrm{A}} \mathrm{d} x\right) \\
& +\operatorname{Re}\left(s_{3} \int_{\Omega}|v|^{2} \mathrm{~A} \Delta \overline{\mathrm{A}} \mathrm{d} x\right) .
\end{aligned}
$$

Adding (3.21) and (3.22) together yields that

$$
\begin{aligned}
& \frac{\mathrm{d}}{\mathrm{dt}}\left(\|\nabla v\|^{2}+\|\nabla \mathrm{A}\|^{2}\right)+2 \alpha\|\Delta v\|^{2}+2 \mu_{1}\|\Delta \mathrm{A}\|^{2} \\
& =2 \mu_{0}\|\nabla \mathrm{A}\|^{2}-2 \beta \mathrm{b}(v, v, \Delta v)-2 \gamma\left(\nabla\left(|\mathrm{A}|^{2}\right), \Delta v\right)-2 \operatorname{Re}\left(s_{1} \int_{\Omega}|v| \mathrm{A} \Delta \overline{\mathrm{A}} \mathrm{d} x\right) \\
& \quad+2 \operatorname{Re}\left(\left(s_{2 r}+i s_{2 i}\right) \int_{\Omega}|\mathrm{A}|^{2} \mathrm{~A} \Delta \overline{\mathrm{A}} \mathrm{d} x\right)+2 \operatorname{Re}\left(s_{3} \int_{\Omega}|v|^{2} \mathrm{~A} \Delta \overline{\mathrm{A}} \mathrm{d} x\right) .
\end{aligned}
$$


Now we need to majorize the right hand side of (3.23). Based on the results in Lemma 3.1, we have

$$
2 \mu_{0}\|\nabla A\|^{2} \leqslant 2 \mu_{0} C\|A\|_{\mathcal{H}^{2}}^{\frac{1}{2}}\|A\|^{\frac{1}{2}} \leqslant \frac{\mu_{1}}{4}\|\Delta A\|^{2}+C .
$$

From the property of $b(u, v, w)$ in (3.11), we obtain

$$
|-2 \beta b(v, v, \Delta v)| \leqslant 2|\beta| \mathrm{C}_{\mathrm{b}}\|v\|^{\frac{1}{2}}\|\nabla v\|\|\Delta v\|^{\frac{3}{2}} \leqslant \frac{\alpha}{4}\|\Delta v\|^{2}+\mathrm{C}_{4}\|\nabla v\|^{4} .
$$

According to the Gagliardo-Nirenberg inequality and Lemma 3.1, we have

$$
\begin{aligned}
\left|-2 \gamma\left(\nabla\left(|A|^{2}\right), \Delta v\right)\right| & \leqslant 4|\gamma|\|A\|_{\mathcal{L}^{\infty}}\|\nabla A\|\|\Delta v\| \\
& \leqslant \frac{\alpha}{4}\|\Delta v\|^{2}+16|\gamma|^{2} \mathrm{C}\|\mathrm{A}\|_{\mathcal{H}^{2}}\|\mathrm{~A}\|\|\nabla \mathrm{A}\|^{2} \\
& \leqslant \frac{\alpha}{4}\|\Delta v\|^{2}+\frac{\mu_{1}}{4}\|\Delta \mathrm{A}\|^{2}+\mathrm{C}_{5}\|\nabla A\|^{4},
\end{aligned}
$$

and

$$
\begin{aligned}
\left|-2 \operatorname{Re}\left(s_{1} \int_{\Omega}|v| \mathrm{A} \Delta \overline{\mathrm{A}} \mathrm{d} x\right)\right| & \leqslant 2\left|s_{1}\right|\|v\|_{\mathbf{L}^{\infty}}\|\mathrm{A}\|\|\Delta \mathrm{A}\| \\
& \leqslant \frac{\mu_{1}}{4}\|\Delta \mathrm{A}\|^{2}+4\left|\mathrm{~s}_{1}\right|^{2} \mathrm{C}\|v\|_{\mathrm{H}^{2}}\|v\|\|\mathrm{A}\|^{2} \\
& \leqslant \frac{\alpha}{4}\|\Delta v\|^{2}+\frac{\mu_{1}}{4}\|\Delta \mathrm{A}\|^{2}+\mathrm{C} .
\end{aligned}
$$

While by virtue of an inequality in [10] and under the condition $\left|s_{2 i}\right|<\sqrt{3} s_{2 r}$, we know that

$$
2 \operatorname{Re}\left(\left(s_{2 r}+i s_{2 i}\right) \int_{\Omega}|A|^{2} A \Delta \bar{A} d x\right) \leqslant 0 .
$$

For the last term in (3.23), we handle it as follows since $\operatorname{Re}\left(s_{3}\right)>0$

$$
\begin{aligned}
2 \operatorname{Re}\left(s_{3} \int_{\Omega}|v|^{2} \mathrm{~A} \Delta \overline{\mathrm{A}} \mathrm{d} x\right) & =-2 \operatorname{Re}\left(s_{3}\right) \int_{\Omega}|v|^{2}|\nabla \mathrm{A}|^{2} \mathrm{~d} x-2 \operatorname{Re}\left(s_{3} \int_{\Omega} \nabla\left(|v|^{2}\right) A \nabla \mathrm{Ad} x\right) \\
& \leqslant-2 \operatorname{Re}\left(\mathrm{s}_{3}\right) \int_{\Omega}|v|^{2}|\nabla \mathrm{A}|^{2} \mathrm{~d} x+4\left|s_{3}\right| \int_{\Omega}|v\|\nabla v\| \mathrm{A} \| \nabla \mathrm{A}| \mathrm{d} x \\
& \leqslant 4\left|s_{3}\right|\|v\|_{\mathbf{L}^{\infty}}\|\mathrm{A}\|_{\mathcal{L}^{\infty}}\|\nabla v\|\|\nabla \mathrm{A}\| \\
& \leqslant 4\left|s_{3}\right| \mathrm{C}\|v\|_{\mathbf{H}^{2}}^{\frac{1}{2}}\|v\|^{\frac{1}{2}}\|\mathrm{~A}\|_{\mathcal{H}^{2}}^{\frac{1}{2}}\|\mathrm{~A}\|^{\frac{1}{2}}\|\nabla v\|\|\nabla \mathrm{A}\| \\
& \leqslant \frac{\alpha}{4}\|\Delta v\|^{2}+\frac{\mu_{1}}{4}\|\Delta \mathrm{A}\|^{2}+\mathrm{C}_{6}\|\nabla v\|^{4}+\mathrm{C}_{7}\|\nabla \mathrm{A}\|^{4} .
\end{aligned}
$$

Combining (3.23)-(3.29), we have

$$
\begin{aligned}
\frac{\mathrm{d}}{\mathrm{dt}}\left(\|\nabla v\|^{2}+\|\nabla \mathrm{A}\|^{2}\right)+\alpha\|\Delta v\|^{2}+\mu_{1}\|\Delta \mathrm{A}\|^{2} & \leqslant\left(\mathrm{C}_{4}+\mathrm{C}_{6}\right)\|\nabla v\|^{4}+\left(\mathrm{C}_{5}+\mathrm{C}_{7}\right)\|\nabla \mathrm{A}\|^{4}+\mathrm{C}_{8} \\
& \leqslant \mathrm{C}_{9}\left(\|\nabla v\|^{2}+\|\nabla \mathrm{A}\|^{2}\right)^{2}+\mathrm{C}_{8},
\end{aligned}
$$

where $C_{9}=C_{4}+C_{5}+C_{6}+C_{7}$ and $C_{8}$ are positive constants depending on the known parameters and $\left\|v_{0}\right\|,\left\|A_{0}\right\|$.

A priori estimates of $v$ in $L^{\infty}\left(0, T ; H^{1}(\Omega)\right)$ and $A$ in $L^{\infty}\left(0, T ; \mathcal{H}^{1}(\Omega)\right)$, for all $T>0$, follow easily from (3.30) by application of the classical Gronwall lemma, using the previous estimates. We are more interested in estimates valid for large $t$, then we apply the uniform Gronwall lemma (Lemma 2.2) to (3.30) with $y, g$, $h$ replaced by

$$
\|\nabla v\|^{2}+\|\nabla A\|^{2}, \quad C_{9}\left(\|\nabla v\|^{2}+\|\nabla A\|^{2}\right), \quad C_{8} .
$$


Thanks to the estimates in Lemma 3.1, we estimate the quantities $a_{1}, a_{2}, a_{3}$ in Lemma 2.2 by $a_{1}=C_{9} a_{3}$, $a_{2}=C_{8} r$, and $a_{3}=\frac{|\gamma|^{2}}{\alpha^{2} C_{3}}\left(C_{2} r+\left\|A_{0}\right\|^{2}+C_{2}\right)+\frac{1}{\alpha}\left(\left\|v_{0}\right\|^{2}+\frac{|\gamma|^{2}}{s_{2 r}}\left\|A_{0}\right\|^{2}\right)+\frac{2|\gamma|^{2} C_{2}}{\alpha^{2} \theta s_{2 r}}+\frac{1}{C_{3}}\left(C_{2} r+\left\|A_{0}\right\|^{2}+C_{2}\right)$. Then we obtain

$$
\|\nabla v\|^{2}+\|\nabla A\|^{2} \leqslant\left(\frac{a_{3}}{r}+a_{2}\right) e^{a_{1}}, \quad \text { for } t \geqslant t_{0}+r,
$$

and $t_{0}$ as in (3.19). This completes the proof of Lemma 3.2.

Lemma 3.3. Assume $v_{0}(x) \in \mathbf{H}_{\text {per }}^{2}(\Omega), A_{0}(x) \in \mathcal{H}_{\mathrm{per}}^{2}(\Omega)$, and the conditions in Lemma 3.2 hold. Then for the solutions of the problem (1.4)-(1.9), we have

$$
\|\Delta v\|^{2}+\|\Delta \mathrm{A}\|^{2} \leqslant\left(\left\|\Delta v_{0}\right\|^{2}+\left\|\Delta \mathrm{A}_{0}\right\|^{2}\right) e^{-\mathrm{t}}+\mathrm{C}\left(1-e^{-\mathrm{t}}\right),
$$

and

$$
\int_{t}^{t+r}\|\nabla \Delta v\|^{2} \mathrm{ds}+\int_{t}^{t+r}\|\nabla \Delta \mathrm{A}\|^{2} \mathrm{ds} \leqslant \mathrm{C}
$$

where $\mathrm{C}$ is a positive constant.

Proof. We take the inner product of (1.4) with $\Delta^{2} v$ in $\mathbf{L}^{2}(\Omega)$ to have

$$
\frac{1}{2} \frac{\mathrm{d}}{\mathrm{dt}}\|\Delta v\|^{2}+\alpha\|\nabla \Delta v\|^{2}=\beta \mathrm{b}\left(v, v, \Delta^{2} v\right)+\gamma\left(\nabla\left(|\mathrm{A}|^{2}\right), \Delta^{2} v\right) .
$$

Multiplying (1.5) by $\Delta^{2} \bar{A}$, integrating with respect to $x$ over $\Omega$ and taking the real part, we obtain that

$$
\begin{aligned}
\frac{1}{2} \frac{\mathrm{d}}{\mathrm{dt}}\|\Delta \mathrm{A}\|^{2}+\mu_{1}\|\nabla \Delta \mathrm{A}\|^{2}= & \mu_{0}\|\Delta \mathrm{A}\|^{2}+\operatorname{Re}\left(\mathrm{s}_{1} \int_{\Omega}|v| \mathrm{A} \Delta^{2} \overline{\mathrm{A}} \mathrm{d} x\right)-\operatorname{Re}\left(s_{2} \int_{\Omega}|\mathrm{A}|^{2} \mathrm{~A} \Delta^{2} \overline{\mathrm{A}} \mathrm{d} x\right) \\
& -\operatorname{Re}\left(s_{3} \int_{\Omega}|v|^{2} \mathrm{~A} \Delta^{2} \overline{\mathrm{A}} \mathrm{d} x\right) .
\end{aligned}
$$

Adding (3.33) and (3.34) together yields that

$$
\begin{aligned}
\frac{\mathrm{d}}{\mathrm{dt}}\left(\|\Delta v\|^{2}+\|\Delta \mathrm{A}\|^{2}\right)+2 \alpha\|\nabla \Delta v\|^{2}+2 \mu_{1}\|\nabla \Delta \mathrm{A}\|^{2} \\
=2 \mu_{0}\|\Delta \mathrm{A}\|^{2}+2 \beta \mathrm{b}\left(v, v, \Delta^{2} v\right)+2 \gamma\left(\nabla\left(|\mathrm{A}|^{2}\right), \Delta^{2} v\right) \\
\quad+2 \operatorname{Re}\left(\mathrm{s}_{1} \int_{\Omega}|v| \mathrm{A} \Delta^{2} \overline{\mathrm{A}} \mathrm{d} x\right)-2 \operatorname{Re}\left(s_{2} \int_{\Omega}|\mathrm{A}|^{2} \mathrm{~A} \Delta^{2} \overline{\mathrm{A}} \mathrm{d} x\right)-2 \operatorname{Re}\left(s_{3} \int_{\Omega}|v|^{2} \mathrm{~A} \Delta^{2} \overline{\mathrm{A}} \mathrm{d} x\right) .
\end{aligned}
$$

From Gagliardo-Nirenberg inequality and previous lemmas, there holds

$$
2 \mu_{0}\|\Delta \mathrm{A}\|^{2} \leqslant 2 \mu_{0} C\|A\|_{\mathcal{H}^{3}}^{\frac{2}{3}}\|\mathrm{~A}\|^{\frac{1}{3}} \leqslant \frac{\mu_{1}}{5}\|\nabla \Delta \mathrm{A}\|^{2}+\mathrm{C} .
$$

While according the definition (3.10), we have

$$
\begin{aligned}
\left|-2 \beta b\left(v, v, \Delta^{2} v\right)\right| & =\left|-2 \beta \int_{\Omega}\left(v_{1} v_{1 x} \Delta^{2} v_{1}+v_{2} v_{1 y} \Delta^{2} v_{1}+v_{1} v_{2 x} \Delta^{2} v_{2}+v_{2} v_{2 y} \Delta^{2} v_{2}\right) \mathrm{d} x \mathrm{~d} y\right| \\
& \leqslant \mathrm{C} \int_{\Omega}\left(|\nabla v|^{2}+|v \| \Delta v|\right)|\nabla \Delta v| \mathrm{d} x \\
& \leqslant \mathrm{C}\left(\|\nabla v\|_{\mathbf{L}^{\infty}}\|\nabla v\|+\|v\|_{\mathbf{L}^{4}}\|\Delta v\|_{\mathbf{L}^{4}}\right)\|\nabla \Delta v\| \\
& \leqslant \mathrm{C}\left(\|v\|_{\mathbf{H}^{3}}^{\frac{2}{3}}\|v\|^{\frac{1}{3}}\|\nabla v\|+\|v\|_{\mathbf{H}^{1}}^{\frac{1}{2}}\|v\|^{\frac{1}{2}}\|v\|_{\mathbf{H}^{3}}^{\frac{5}{6}}\|v\|^{\frac{1}{6}}\right)\|\nabla \Delta v\| \\
& \leqslant \frac{\alpha}{4}\|\nabla \Delta v\|^{2}+\mathrm{C} .
\end{aligned}
$$

In the same way, by the Gagliardo-Nirenberg inequality and previous results, we obtain the following 
estimates

$$
\begin{aligned}
& \left|2 \gamma\left(\nabla\left(|A|^{2}\right), \Delta^{2} v\right)\right| \leqslant 4|\gamma| \int_{\Omega}\left(|\Delta \mathrm{A}||\mathrm{A}|+|\nabla A|^{2}\right)|\nabla \Delta v| \mathrm{d} x \\
& \leqslant 4|\gamma|\left(\|\Delta A\|_{\mathcal{L}^{4}}\|A\|_{\mathcal{L}^{4}}+\|\nabla A\|_{\left.\mathcal{L}^{\infty}\|\nabla A\|\right)\|\nabla \Delta v\|}\right. \\
& \leqslant \mathrm{C}\left(\|\mathrm{A}\|_{\mathcal{H}^{3}}^{\frac{5}{6}}\|\mathrm{~A}\|^{\frac{1}{6}}\|\mathrm{~A}\|_{\mathcal{H}^{1}}^{\frac{1}{2}}\|\mathrm{~A}\|^{\frac{1}{2}}+\|\mathrm{A}\|_{\mathcal{H}^{3}}^{\frac{2}{3}}\|\mathrm{~A}\|^{\frac{1}{3}}\|\nabla A\|\right)\|\nabla \Delta v\| \\
& \leqslant \frac{\alpha}{4}\|\nabla \Delta v\|^{2}+\frac{\mu_{1}}{5}\|\nabla \Delta A\|^{2}+C, \\
& \left|2 \operatorname{Re}\left(s_{1} \int_{\Omega}|v| A \Delta^{2} \bar{A} d x\right)\right| \leqslant 2\left|s_{1}\right|\left(\|A\|_{\mathcal{L}^{\infty}}\|\nabla v\|+\|v\|_{\mathbf{L}^{\infty}}\|\nabla A\|\right)\|\nabla \Delta A\| \\
& \leqslant 2\left|s_{1}\right| C\|A\|_{\mathcal{H}^{3}}^{\frac{1}{3}}\|A\|^{\frac{2}{3}}\|\nabla v\|\|\nabla \Delta A\| \\
& +2\left|s_{1}\right| \mathrm{C}\|v\|_{\mathrm{H}^{3}}^{\frac{1}{3}}\|v\|^{\frac{2}{3}}\|\nabla \mathrm{A}\|\|\nabla \Delta \mathrm{A}\| \\
& \leqslant \frac{\alpha}{4}\|\nabla \Delta v\|^{2}+\frac{\mu_{1}}{5}\|\nabla \Delta A\|^{2}+C \text {, }
\end{aligned}
$$

and

$$
\begin{aligned}
\left|-2 \operatorname{Re}\left(s_{2} \int_{\Omega}|\mathrm{A}|^{2} \mathrm{~A} \Delta^{2} \overline{\mathrm{A}} \mathrm{d} x\right)\right| & \leqslant 6\left|s_{2}\right|\|\mathrm{A}\|_{\mathcal{L}^{\infty}}^{2}\|\nabla \mathrm{A}\|\|\nabla \Delta \mathrm{A}\| \\
& \leqslant 6\left|s_{2}\right| \mathrm{C}\|\mathrm{A}\|_{\mathcal{F}^{3}}^{\frac{2}{3}}\|\mathrm{~A}\|\left\|^{\frac{4}{3}}\right\| \nabla \mathrm{A}\|\| \nabla \Delta \mathrm{A} \| \\
& \leqslant \frac{\mu_{1}}{5}\|\nabla \Delta \mathrm{A}\|^{2}+\mathrm{C} .
\end{aligned}
$$

It is easy to handle the last term as follows

$$
\begin{aligned}
\left|-2 \operatorname{Re}\left(s_{3} \int_{\Omega}|v|^{2} A \Delta^{2} \bar{A} d x\right)\right| & \leqslant 2\left|s_{3}\right|\left(2\|v\|_{\mathbf{L}^{\infty}}\|A\|_{\mathcal{L}^{\infty}}\|\nabla v\|+\|v\|_{\mathbf{L}^{\infty}}^{2}\|\nabla A\|\right)\|\nabla \Delta A\| \\
& \leqslant C\left(\|v\|_{\mathbf{H}^{3}}^{\frac{1}{3}}\|v\|^{\frac{2}{3}}\|A\|_{\mathcal{H}^{3}}^{\frac{1}{3}}\|A\|^{\frac{2}{3}}+\|v\|_{\mathbf{H}^{3}}^{\frac{2}{3}}\|v\|^{\frac{4}{3}}\right)\|\nabla \Delta A\| \\
& \leqslant \frac{\alpha}{4}\|\nabla \Delta v\|^{2}+\frac{\mu_{1}}{5}\|\nabla \Delta A\|^{2}+C .
\end{aligned}
$$

Then substituting (3.36)-(3.41) into (3.35), there arrives

$$
\frac{\mathrm{d}}{\mathrm{dt}}\left(\|\Delta v\|^{2}+\|\Delta \mathrm{A}\|^{2}\right)+\alpha\|\nabla \Delta v\|^{2}+\mu_{1}\|\nabla \Delta \mathrm{A}\|^{2} \leqslant \mathrm{C}
$$

Noticing that $\|\Delta v\|^{2} \leqslant \alpha\|\nabla \Delta v\|^{2}+\mathrm{C}$ and $\|\Delta \mathrm{A}\|^{2} \leqslant \mu_{1}\|\nabla \Delta \mathrm{A}\|^{2}+\mathrm{C}$, thus there holds

$$
\frac{\mathrm{d}}{\mathrm{dt}}\left(\|\Delta v\|^{2}+\|\Delta \mathrm{A}\|^{2}\right)+\|\Delta v\|^{2}+\|\Delta \mathrm{A}\|^{2} \leqslant \mathrm{C} .
$$

Applying the Gronwall's inequality concludes (3.31). Finally integrating in $t$ in (3.42), we have (3.32). Thus the proof of Lemma 3.3 is completed.

Generally based on the results of the previous lemmas and the mathematical deduction, we have the following lemma for problem (1.4)-(1.9).

Lemma 3.4. Assume $v_{0}(x) \in \mathbf{H}_{\mathrm{per}}^{\mathrm{k}}(\Omega), \mathrm{A}_{0}(x) \in \mathcal{H}_{\mathrm{per}}^{\mathrm{k}}(\Omega)(\mathrm{k} \geqslant 3)$, and the conditions (1.10) hold. Then for the solutions of the problem (1.4)-(1.9), we have

$$
\|v\|_{\mathbf{H}^{k}}^{2}+\|A\|_{\mathcal{H}^{k}}^{2} \leqslant C,
$$

where $\mathrm{C}$ is a positive constant depending on the known parameters and $\left\|v_{0}\right\|_{\mathbf{H}^{\mathrm{k}}},\left\|\mathrm{A}_{0}\right\|_{\mathcal{H}^{\mathrm{k}}}$.

\section{The local solutions and global solutions}

In this section, we will obtain the existence and uniqueness of the local solutions and global solutions for the periodic initial value problem (1.4)-(1.9). Firstly, we adopt the Galerkin method to construct the approximate solutions for the problem (1.4)-(1.9). Let $\omega_{j}(x)(j=1,2, \cdots)$ be the unit eigenfunctions satisfying the equation

$$
\Delta \omega_{j}+\lambda_{j} \omega_{j}=0, \quad j=1,2, \cdots, \quad \omega_{j} \in H_{0}^{1}(\Omega) \cap L^{4}(\Omega),
$$


with periodicity $\omega_{j}(x)=\omega_{j}\left(x+\operatorname{Le} e_{i}\right)(i=1,2)$ and $\lambda_{j}(j=1,2, \cdots)$ is the corresponding eigenvalues different from each other $\left\{\omega_{j}(x)\right\}$ consists of the orthogonal base in $\mathrm{L}^{2}(\Omega)$. Thus the approximate solutions can be written as

$$
v_{m}(x, t)=\sum_{j=1}^{m} g_{j m}(t) \omega_{j}(x), \quad A_{m}(x, t)=\sum_{j=1}^{m} h_{j m}(t) \omega_{j}(x) .
$$

According to the Galerkin method, these undetermined coefficients $g_{j m}(t)$ and $h_{j m}(t)$ have to satisfy the following initial value problem of a system of the ordinary differential equations

$$
\begin{aligned}
\left(v_{m t}, \omega_{j}\right)= & \alpha\left(\Delta v_{m}, \omega_{j}\right)+\beta\left(\left(v_{m} \cdot \nabla\right) v_{m}, \omega_{j}\right)+\gamma\left(\nabla\left(\left|A_{m}\right|^{2}\right), \omega_{j}\right), \\
\left(A_{m t}, \omega_{j}\right)= & \mu_{0}\left(A_{m}, \omega_{j}\right)+\left(\mu_{1}+i \mu_{2}\right)\left(\Delta A_{m}, \omega_{j}\right)+s_{1}\left(\left|v_{m}\right| A_{m}, \omega_{j}\right) \\
& -s_{2}\left(\left|A_{m}\right|^{2} A_{m}, \omega_{j}\right)-s_{3}\left(\left|v_{m}\right|^{2} A_{m}, \omega_{j}\right),
\end{aligned}
$$

with initial conditions

$$
v_{m}(x, 0)=v_{0 m}(x), \quad A_{m}(x, 0)=A_{0 m}(x),
$$

where $0 \leqslant t \leqslant T$ and $j=1,2, \cdots, m$.

We assume that

$$
v_{0 \mathrm{~m}}(\mathrm{x}) \stackrel{\mathrm{H}_{\mathrm{per}}^{2}(\Omega)}{\longrightarrow} v_{0}(\mathrm{x}), \quad \mathrm{A}_{0 \mathrm{~m}}(\mathrm{x}) \stackrel{\mathcal{H}_{\mathrm{per}}^{2}(\Omega)}{\longrightarrow} \mathrm{A}_{0}(\mathrm{x}), \quad \mathrm{m} \rightarrow \infty .
$$

Similar to the proof of Lemmas 3.1, 3.2 and 3.3, we can establish the estimates of the solutions of the problem (1.4)-(1.9) which are uniform for $\mathrm{m}$. By using the compact principle, we can prove the following.

Theorem 4.1 (Local existence). Assume that $v_{0}(x) \in \mathbf{H}_{\mathrm{per}}^{2}(\Omega), \mathrm{A}_{0}(x) \in \mathcal{H}_{\mathrm{per}}^{2}(\Omega)$, and the conditions (1.10) hold. Then the periodic initial value problem (1.4)-(1.9) possesses the periodic local solutions $v(x, t)$ and $A(x, t)$, which satisfy

$$
\begin{array}{cl}
v(x, t) \in L^{\infty}\left(0, t_{0} ; H_{\text {per }}^{2}(\Omega)\right), & v_{t}(x, t) \in L^{\infty}\left(0, t_{0} ; \mathbf{L}_{\text {per }}^{2}(\Omega)\right), \\
A(x, t) \in L^{\infty}\left(0, t_{0} ; \mathcal{H}_{\text {per }}^{2}(\Omega)\right), & A_{t}(x, t) \in L^{\infty}\left(0, t_{0} ; \mathcal{H}_{\text {per }}^{1}(\Omega)\right),
\end{array}
$$

where $\mathrm{t}_{0}$ depends on $\left\|v_{0}(\mathrm{x})\right\|_{\mathrm{H}_{\text {per }}^{2}}$ and $\left\|\mathrm{A}_{0}(\mathrm{x})\right\|_{\mathcal{H}_{\text {per }}^{2}}$.

Theorem 4.2 (Global existence and uniqueness). Suppose the conditions of Theorem 4.1 fulfill. Then there exist unique global solutions $v(x, t)$ and $\mathrm{A}(\mathrm{x}, \mathrm{t})$, which satisfy

$$
\begin{array}{cc}
v(x, t) \in \mathrm{L}^{\infty}\left(0, \mathrm{~T} ; \mathrm{H}_{\text {per }}^{2}(\Omega)\right), & v_{\mathrm{t}}(\mathrm{x}, \mathrm{t}) \in \mathrm{L}^{\infty}\left(0, \mathrm{~T} ; \mathrm{L}_{\text {per }}^{2}(\Omega)\right), \\
\mathrm{A}(\mathrm{x}, \mathrm{t}) \in \mathrm{L}^{\infty}\left(0, \mathrm{~T} ; \mathcal{H}_{\text {per }}^{2}(\Omega)\right), & \mathrm{A}_{\mathrm{t}}(\mathrm{x}, \mathrm{t}) \in \mathrm{L}^{\infty}\left(0, \mathrm{~T} ; \mathcal{L}_{\text {per }}^{2}(\Omega)\right),
\end{array}
$$

for the periodic initial value problem (1.4)-(1.9).

Proof. From Theorem 4.1 we know that the local solutions for the problem (1.4)-(1.9) exist and $t_{0}$ depends on $\left\|v_{0}(x)\right\|_{\mathbf{H}_{\text {per }}^{2}}$ and $\left\|A_{0}(x)\right\|_{\mathcal{H}_{\mathrm{per}}^{2}}$. According to the priori estimates in Section 3 and by the so-called continuity method, we can obtain the global solutions for the problem (1.4)-(1.9) easily.

More generally, we have the following existence and uniqueness theorems of the global smooth solutions from Lemma 3.4.

Theorem 4.3 (Existence and uniqueness for global smooth solutions). Suppose that $v_{0}(x) \in \mathbf{H}_{\text {per }}^{k}(\Omega)$, $A_{0}(x) \in \mathcal{H}_{\text {per }}^{k}(\Omega)(k \geqslant 3)$ and the conditions (1.10) hold. Then there exist unique global smooth solutions $v(x, t)$ and $\mathrm{A}(\mathrm{x}, \mathrm{t})$, which satisfy

$$
\begin{array}{cc}
v(x, t) \in \mathrm{L}^{\infty}\left(0, \mathrm{~T} ; \mathrm{H}_{\text {per }}^{\mathrm{k}}(\Omega)\right), & v_{\mathrm{t}}(\mathrm{x}, \mathrm{t}) \in \mathrm{L}^{\infty}\left(0, \mathrm{~T} ; \mathrm{H}_{\text {per }}^{\mathrm{k}-2}(\Omega)\right), \\
\mathrm{A}(\mathrm{x}, \mathrm{t}) \in \mathrm{L}^{\infty}\left(0, \mathrm{~T} ; \mathcal{H}_{\text {per }}^{\mathrm{k}}(\Omega)\right), & \mathrm{A}_{\mathrm{t}}(\mathrm{x}, \mathrm{t}) \in \mathrm{L}^{\infty}\left(0, \mathrm{~T} ; \mathcal{H}_{\text {per }}^{\mathrm{k}-2}(\Omega)\right),
\end{array}
$$

for the periodic initial value problem (1.4)-(1.9). 


\section{The existence of global attractor}

In this section, we construct the global attractor for the problem (1.4)-(1.9). We first note that by Theorem 4.2, there exists a dynamical system $\mathbf{S}(\mathbf{t})(\mathbf{t} \geqslant 0)$ which maps $\mathbf{H}_{\text {per }}^{2}(\Omega) \times \mathcal{H}_{\text {per }}^{2}(\Omega)$ to $\mathbf{H}_{\text {per }}^{2}(\Omega) \times$ $\mathcal{H}_{\text {per }}^{2}(\Omega)$ such that $S(t)\left(v_{0}, A_{0}\right)=(v(t), A(t))$, the solutions of problem (1.4)-(1.9). Firstly from Lemmas 3.1, 3.2 and 3.3, we have the uniform a priori estimates in time, which implies

$$
\|v(t)\|_{\mathbf{H}_{\text {per }}^{2}}+\|A(t)\|_{\mathcal{H}_{\text {per }}^{2}} \leqslant K, \quad \forall t \geqslant t_{0}
$$

where $\mathrm{K}$ is a positive constant.

In what follows, we are going to show that the semigroup $S(t): \mathbf{H}_{\text {per }}^{2}(\Omega) \times \mathcal{H}_{\text {per }}^{2}(\Omega) \rightarrow \mathbf{H}_{\text {per }}^{2}(\Omega) \times$ $\mathcal{H}_{\text {per }}^{2}(\Omega)$ is compact for large $t$. That is

Lemma 5.1. Assume that the conditions of Theorem 4.2 hold. Then for the solutions of the problem (1.4)-(1.9), we have

$$
\|\nabla \Delta v\|^{2}+\|\nabla \Delta \mathrm{A}\|^{2} \leqslant \mathrm{C}, \quad \forall \mathrm{t} \geqslant \mathrm{t}_{0},
$$

where the constant $\mathrm{C}$ depends on the known parameters and the data $\left\|v_{0}\right\|_{\mathbf{H}_{\mathrm{per}^{2}}^{2}}\left\|A_{0}\right\|_{\mathcal{H}_{\mathrm{per}}^{2}}$.

Proof. Similar to the proofs in previous lemmas, we take the inner product of (1.4) with $\Delta^{3} v$ in $\mathbf{L}^{2}(\Omega)$ and (1.5) with $\Delta^{3} A$ in $\mathcal{L}^{2}(\Omega)$. Adding the two equations together and majorizing each term with previous estimates, we have

$$
\frac{\mathrm{d}}{\mathrm{dt}}\left(\|\nabla \Delta v\|^{2}+\|\nabla \Delta \mathrm{A}\|^{2}\right) \leqslant \mathrm{C}\left(\|\nabla \Delta v\|^{2}+\|\nabla \Delta \mathrm{A}\|^{2}\right)+\mathrm{C} .
$$

Applying (3.32) in Lemma 3.3, integrating (5.2) in $t$ and by the uniform Gronwall lemma, we obtain that

$$
\|\nabla \Delta v\|^{2}+\|\nabla \Delta \mathrm{A}\|^{2} \leqslant \mathrm{C}, \quad \forall \mathrm{t} \geqslant \mathrm{t}_{0},
$$

where the constant $C$ depends on the known parameters and the data $\left\|v_{0}\right\|_{\mathbf{H}_{\mathrm{per}}^{2}}\left\|A_{0}\right\|_{\mathcal{H}_{\mathrm{per}}^{2}}$. Thus the proof of Lemma 5.1 is completed.

In order to prove the existence of global attractor of problem (1.4)-(1.9), we need the following result:

Theorem 5.2 ([18]). We assume that $\mathrm{H}$ is a metric space and that the nonlinear operator $\mathrm{S}(\mathrm{t})$ of $\mathrm{H}$ into itself for $t \geqslant 0$ satisfied

$$
\mathrm{S}(\mathrm{t}+\mathrm{s})=\mathrm{S}(\mathrm{t}) \cdot \mathrm{S}(\mathrm{s}), \quad \forall \mathrm{s}, \mathrm{t} \geqslant 0, \quad \mathrm{~S}(0)=\mathrm{I}, \quad \text { (Identity in } \mathrm{H}) .
$$

And also $\mathrm{S}(\mathrm{t})$ is continuous and uniformly compact for large $\mathrm{t}$. That means for every bounded set $\mathrm{B}$, there exists $\mathrm{t}_{0}$, which may depend on $\mathrm{B}$ that $\bigcup_{\mathrm{t} \geqslant \mathrm{t}_{0}} \mathrm{~S}(\mathrm{t}) \mathrm{B}$ is relatively compact in $\mathrm{H}$. We also assume that there exists an open set $\mathrm{U}$ and a bounded set $\mathrm{B}$ of $\mathrm{U}$ such that $\mathrm{B}$ is absorbing in $\mathrm{U}$.

Then the $\omega$-limit set of $\mathrm{B}: \mathcal{A}=\omega(\mathrm{B})=\bigcap_{\mathrm{s} \geqslant 0} \bigcup_{\mathrm{t} \geqslant \mathrm{s}} \mathrm{S}(\mathrm{t}) \mathrm{B}$ is a compact attractor, which attracts the bounded set of $\mathrm{U}$. It is the maximal bounded attractor in $\mathrm{U}$.

Theorem 5.3. Assume that the conditions of Theorem 4.2 hold. Then there exists a global attractor $\mathcal{A} \subset \mathbf{H}_{\text {per }}^{2}(\Omega) \times$ $\mathcal{H}_{\text {per }}^{2}(\Omega)$ for the periodic initial problem (1.4)-(1.9), i.e., there is a set $\mathcal{A}$ such that

(1) $\mathrm{S}(\mathrm{t}) \mathcal{A}=\mathcal{A}, \mathrm{t} \in \mathrm{R}^{+}$;

(2) $\lim _{\mathrm{t} \rightarrow \infty} \operatorname{dist}(\mathrm{S}(\mathrm{t}) \mathrm{B}, \mathcal{A})=0$, for any bounded set $\mathrm{B} \subset \mathbf{H}_{\mathrm{per}}^{2}(\Omega) \times \mathcal{H}_{\mathrm{per}}^{2}(\Omega)$, where

$$
\operatorname{dist}(X, Y)=\sup _{x \in X} \inf _{y \in Y}\|x-y\|_{E}
$$

and $\mathrm{S}(\mathrm{t})\left(v_{0}, \mathrm{~A}_{0}\right)$ is a semigroup operator generated by the problem (1.4)-(1.9). 
Proof. On account of the result of Theorem 5.2, we will prove this theorem by checking the conditions in Theorem 5.2. We observe that (5.1) shows that the ball

$$
\mathrm{B}=\left\{(v, \mathrm{~A}) \in \mathbf{H}_{\mathrm{per}}^{2}(\Omega) \times \mathcal{H}_{\mathrm{per}}^{2}(\Omega):\|v(\mathrm{t})\|_{\mathbf{H}_{\mathrm{per}}^{2}} \leqslant \mathrm{~K},\|\mathrm{~A}(\mathrm{t})\|_{\mathcal{H}_{\mathrm{per}}^{2}} \leqslant K\right\}
$$

is an absorbing set of $S(t)$ in $\mathbf{H}_{\text {per }}^{2}(\Omega) \times \mathcal{H}_{\text {per }}^{2}(\Omega)$. In addition, Lemma 5.1 implies the dynamical system $S(t)$ is uniformly compact for large $t$. Thus, according to Theorem 5.2, we can conclude that the $\omega$-limit set of $B: \mathcal{A}=\omega(B)=\bigcap_{s \geqslant 0} \overline{\bigcup_{t} \geqslant s} \mathrm{~S}(\mathrm{t}) \mathrm{B}$ is a compact attractor on $\mathbf{H}_{\text {per }}^{2}(\Omega) \times \mathcal{H}_{\text {per }}^{2}(\Omega)$, where the closure is taken in $\mathbf{H}_{\text {per }}^{2}(\Omega) \times \mathcal{H}_{\text {per }}^{2}(\Omega)$. This completes the proof of Theorem 5.3.

Generally by induction and the estimates in Lemma 3.4, we have the following result.

Theorem 5.4. The semigroup of the nonlinear operators $\{\mathrm{S}(\mathrm{t})\}$ determined by the periodic initial problem (1.4)-(1.9) has a compact connect global attractor $\mathcal{A}$ in $\mathbf{H}_{\mathrm{per}}^{\mathrm{k}}(\Omega) \times \mathcal{H}_{\mathrm{per}}^{\mathrm{k}}(\Omega)$, which attracts all bounded sets of $\mathbf{H}_{\mathrm{per}}^{\mathrm{k}}(\Omega) \times$ $\mathcal{H}_{\mathrm{per}}^{\mathrm{k}}(\Omega)$, for all $\mathrm{k} \geqslant 0$.

\section{Acknowledgment}

This research was supported by the National Natural Science Foundation of China (No. 11426069, 11271141), and Natural Science Foundation of Guangdong Province, China (No. 2014A030313514).

\section{References}

[1] A. J. S. Al-Saif, A. Abdul-Hussein, Generating exact solutions of two-dimensional coupled Burgers equations by the first integral method, Res. J. Phys. Appl. Sci., 1 (2012), 29-33. 1

[2] A. R. Bahadır, A fully implicit finite-difference scheme for two-dimensional Burgers' equations, Appl. Math. Comput., 137 (2003), 131-137. 1

[3] A. J. Bernoff, Slowly varying fully nonlinear wavetrains in the Ginzburg-Landau equation, Phys. D, 30 (1988), $363-381$. 1

[4] J. M. Burgers, A mathematical model illustrating the theory of turbulence, edited by Richard von Mises and Theodore von Kármán, Advances in Applied Mechanics, Academic Press, Inc., New York, N. Y., (1948), 171-199. 1

[5] A. Friedman, Partial differential equations, Holt, Rinehart and Winston, Inc., New York-Montreal, Que.-London, (1969). 2.1

[6] C.-H. Guo, S.-M. Fang, B.-L. Guo, Global smooth solutions of the generalized KS-CGL equations with flames governed by a sequential reaction, Commun. Math. Sci., 12 (2014), 1457-1474. 1

[7] C.-H. Guo, S.-M. Fang, B.-L. Guo, Long time behavior of solutions to coupled Burgers-complex Ginzbury-Landau (Burgers-CGL) equations for flames governed by sequential reaction, [[Long time behavior of solutions to coupled Burgers-complex Ginzburg-Landau (Burgers-CGL) equations for flames governed by sequential reaction]] Appl. Math. Mech. (English Ed.), 35 (2014), 515-534. 1

[8] B.-L. Guo, H.-Y. Huang, On the Burgers-Ginzburg-Landau equations, Commun. Nonlinear Sci. Numer. Simul., 3 (1998), 143-147. 1

[9] B.-L. Guo, H.-Y. Huang, Well-posedness and global attractors for the Burgers-Ginzburg-Landau equations, Acta Math. Appl. Sin. Engl. Ser., 18 (2002), 579-588. 1

[10] D. Henry, Geometric theory of semilinear parabolic equations, Lecture Notes in Mathematics, Springer-Verlag, BerlinNew York, (1981). 3

[11] H.-Y. Huang, Attractors of the discretized Burgers-Ginzburg-Landau equations, (Chinese) Acta Math. Sci. Ser. A Chin. Ed., 22 (2002), 316-322. 1

[12] H.-Y. Huang, Existence of periodic solutions of the Burgers-Ginzburg-Landau equations, Acta Math. Sci. Ser. B Engl. Ed., 24 (2004), 613-622. 1

[13] R. Iturriaga, K. Khanin, Burgers turbulence and random Lagrangian systems, Comm. Math. Phys., 232 (2003), 377-428. 1

[14] L. Kramer, W. Zimmermann, On the Eckhaus instability for spatially periodic patterns, Phys. D, 16 (1985), 221-232. 1

[15] A. Mielke, G. Schneider, Derivation and justification of the complex Ginzburg-Landau equation as a modulation equation, Dynamical systems and probabilistic methods in partial differential equations, Berkeley, CA, (1994), Lectures in Appl. Math., Amer. Math. Soc., Providence, RI, 31 (1996), 191-216. 1 
[16] A. A. Nepomnhyashchy, Order parameter equations for long-wavelength instabilities, Phys. D, 86 (1995), 90-95. 1

[17] K. Nishinari, J. Matsukidaira, D. Takahashi, Two-dimensional Burgers cellular automaton, J. Phys. Soc. Japan, 70 (2001), 2267-2272. 1, 1

[18] R. Temam, Infinite-dimensional dynamical systems in mechanics and physics, Second edition, Applied Mathematical Sciences, Springer-Verlag, New York, (1997). 2.2, 3, 5.2

[19] A. van Harten, Modulated modulation equations, Proceedings of the IUTAM/ISIMM Symposium on Structure and Dynamics of Nonlinear Waves in Fluids, Hannover, (1994), Adv. Ser. Nonlinear Dynam., World Sci. Publ., River Edge, NJ, 7 (1995), 117-130. 1

[20] H.-Q. Zhu, H.-Z. Shu, M.-Y. Ding, Numerical solutions of two-dimensional Burgers' equations by discrete Adomian decomposition method, Comput. Math. Appl., 60 (2010), 840-848. 1 